\title{
Avaliação dos potenciais antioxidante e tóxico de amidas aromáticas sintetizadas seguindo a Química Verde
}

\author{
Evaluation of antioxidant and toxic potentials of aromatic amides synthesized following Green \\ Chemistry \\ Evaluación del potencial antioxidante y tóxico de las amidas aromáticas sintetizadas siguiendo la \\ Química Verde
}

Kelly Clair de Moura da Costa ORCID: https://orcid.org/0000-0003-3483-5017 Universidade Federal de Mato Grosso, Brasil E-mail: kellyclair.farm@gmail.com

Danúbia Jussara Bento da Silva ORCID: https://orcid.org/0000-0001-7587-7198 Universidade Federal de Mato Grosso, Brasil E-mail: danubiajussara@gmail.com

Jair Marques Junior ORCID: https://orcid.org/0000-0002-0949-1139 Gerência de Medicina Legal Paul Harris, Brasil E-mail: jairmarquesjunior@yahoo.com.br

Claudemir Batalini

ORCID: https://orcid.org/0000-0002-5915-0850 Universidade Federal de Mato Grosso, Brasil E-mail: pirapotimao@msn.com

\begin{abstract}
Resumo
A síntese orgânica corresponde ao maior meio de produção de novos fármacos na terapêutica atualmente disponível no mercado, desse modo, pensar em processos de produção que visem a menor agressão ao meio ambiente e geração de resíduos é imprescindível, principalmente de amidas aromáticas que por via clássica de síntese geram resíduos, e são amplamente aplicadas na indústria química e farmacêutica de modo geral. Este estudo analisou o padrão antioxidante e tóxico de cinco amidas aromáticas sintetizadas seguindo preceitos da Química Verde - pelo método de Schotten-Baumann, sendo enaltecida sua relação estrutura-atividade para explicar os padrões encontrados no estudo. Ressalta-se que duas delas apresentaram resultados promissores para o teste antioxidante e tóxico, e podem seguir adiante em estudos futuros que testem com segurança suas aplicações e usos como compostos bioativos, ambas são consideradas inéditas na literatura até o presente momento, estabelecendo, portanto, o importante vínculo entre as vias alternativas verdes de produção para o meio industrial e o meio ambiente.
\end{abstract}

Palavras-chave: Química verde; Amidas aromáticas; Potencial tóxico; Potencial antioxidante; Método de SchottenBaumann.

\begin{abstract}
Organic synthesis corresponds to the largest means of production of new drugs in therapeutics currently available on the market, thus, thinking about production processes that aim at less harm to the environment and generation of waste is essential, especially of aromatic amides that are classically synthesis products generate waste, and are widely applied in the chemical and pharmaceutical industry in general. This study analyzed the antioxidant and toxic potential of five aromatic amides synthesized following the precepts of Green Chemistry - by the Schotten-Baumann method, and their structure-activity relationship was highlighted to explain the patterns found in the study. We emphasize that two of them showed promising results for the antioxidant and toxic test, and may go ahead in future studies that safely test their applications and uses as bioactive compounds, and it is noteworthy that both are considered unprecedented in the literature to date, establishing, therefore, the important link between alternative green production routes for the industrial mode and the environment.
\end{abstract}

Keywords: Green chemistry; Aromatic amides; Toxic potential; Antioxidant potential; Schotten-Baumann Method.

\section{Resumen}

La síntesis orgánica corresponde al mayor medio de producción de nuevos fármacos en la terapia actualmente disponibles en el mercado, por lo que es fundamental pensar en procesos de producción que apunten a la menor agresión al medio ambiente y generación de residuos, principalmente de amidas aromáticas que por vía clásica vía de 
síntesis generan residuos, y son de amplia aplicación en la industria química y farmacéutica en general. Este estudio analizó el patrón antioxidante y tóxico de cinco amidas aromáticas sintetizadas siguiendo los preceptos de la Química Verde, por el método de Schotten-Baumann, destacando su relación estructura-actividad para explicar los patrones encontrados en el estudio. Resaltamos que dos de ellos mostraron resultados prometedores para la prueba de antioxidantes y tóxicos, y pueden salir adelante en futuros estudios que prueben con seguridad sus aplicaciones y usos como compuestos bioactivos, y cabe mencionar que ambos se consideran inéditos en la literatura a la fecha, estableciendo, por tanto, el importante vínculo entre las formas alternativas verdes de producción para el entorno industrial y el medio ambiente.

Palabras clave: Química verde; Amidas aromáticas; Potencial tóxico; Potencial antioxidante; Método de SchottenBaumann.

\section{Introdução}

Cresce a demanda por substâncias bioativas que apresentem diversas aplicações para a indústria química e farmacêutica e possuam vias sintéticas mais simples, menos agressivas ao meio ambiente e com baixa toxicidade para o ser humano. As amidas são compostos orgânicos comumente produzidos por rotas sintéticas, apresentam diversas propriedades físico-químicas as quais garantem sua versatilidade em diversos produtos e fazem parte de um amplo espectro de fármacos desenvolvidos atualmente. Desse modo, buscar novas rotas que sejam de baixo custo e favoráveis ao meio ambiente é o que a Química Verde se propõe (Mãrino, 2014; Calvalcante et al., 2015; Barreiro \& Fraga, 2015).

O conceito de Química Verde (tradução livre de "Green Chemistry") foi desenvolvido como modelo autossustentável que aborda 12 princípios os quais garantem a redução de poluentes e dejetos, promovendo menos consumo de energia e agressão ao ambiente. É uma tecnologia limpa que avança nos laboratórios das grandes indústrias e marcas do mundo, mediante a urgente demanda em proteger o ambiente e ainda assim continuar produzindo, saber conciliar o processo de produção e a questão ambiental e a necessidade do momento (Lenardão et al., 2003; Anastas \& Eghbali, 2010; Zimmerman et al., 2020).

Apesar de amplamente conhecidas, as amidas aromáticas ainda são bastante estudadas na Química-Farmacêutica devido a grande aplicação que possuem; novas conformações moleculares, interações e modificações estruturais fornecem uma gama de substâncias inéditas ou pouco exploradas até o momento e a síntese verde tem um papel crucial nesse âmbito. Assim, o método de Schotten-Baumann parte do pressuposto de que cloretos de ácido aromático podem combinar-se com fenóis e aminas aromáticas para a produção de ésteres e amidas aromáticas e essa reação segue pelo menos 6 princípios básicos da química verde, sendo eles: meio aquoso básico menos agressivo, não dependem de catálise, não formam derivados pois são reações diretas, usam pouca energia porque a temperatura para reação ocorrer é ambiente, geram poucos resíduos e necessitam de reagentes menos agressivos (Schotten, 1884; Baumann, 1886; Solomons et al., 2014).

Outro fator importante para a aplicabilidade de um futuro composto bioativo é conhecer seus perfis de toxicidade e a sua capacidade antioxidante. Essas análises são as primeiras de um completo rol de estudos que devem preceder a eficácia e viabilidade da substância em questão. O potencial tóxico, em linhas gerais, trata-se da capacidade de um composto em causar dano a uma célula e o potencial antioxidante determina se a substância é capaz de absorver ou neutralizar radicais livres. Sabese que algumas estruturas químicas derivadas do nitrogênio e do benzeno possuem perfil tóxico para o ser humano e meio ambiente, desse modo, estudar substâncias que possuem em sua estrutura esses grupamentos químicos é sempre necessário e inerente antes de pensar sua possível aplicação (Barbosa et al., 2010; Medeiros et al., 2020).

Desse modo, esta pesquisa baseou-se na investigação do potencial antioxidante e tóxico de cinco amidas aromáticas (Figura 1), sendo que duas delas ainda não foram encontradas descritas na literatura; todas foram sintetizadas previamente pelo método de Schotten-Baumann que segue preceitos da Química Verde no Laboratório de Química de Produtos Naturais (LAPQUÍM), localizado na Universidade Federal de Mato - UFMT, Campus Universitário do Araguaia. 


\section{Metodologia}

\subsection{Investigação do potencial antioxidante}

Para o teste do potencial antioxidante das substâncias (I) a (V) foi empregado o método qualitativo de DPPH desenvolvido por Brand-Willams e colaboradores (1995) (apud: Rockenbach, 2008). Cada amida foi analisada por CCD (Cromatografia em Camada Delgada), usando rutina (P.A. - Sigma Aldrich) como padrão positivo de comparação. As placas foram eluídas em solvente diclorometano:metanol a 10\% acrescentando-se então cada amida de partida e após secagem das placas, foi observada cada uma em câmara de ultravioleta-visível e a seguir nebulizadas com solução 0,4 mmol.L $\mathrm{L}^{-1}$ do radical DPPH (difenilpicrilhidrazil) em metanol, para observação do aparecimento de manchas amarelas sob o fundo de coloração púrpura, indicativo de possível atividade antioxidante (Sousa et al., 2007).

Os valores de fator de retenção (FR) de cada substância e do padrão foram calculados na placa cromatográfica pela relação da distância da origem da eluição até o centro da mancha dividido pela distância da origem até o final da corrida do eluente.

\subsection{Investigação do potencial tóxico}

A avaliação do potencial tóxico das amidas (I) a (V) foi realizada frente às larvas de Artemia salina Leach, seguindose procedimentos de Meyer et al. (1982), com algumas modificações, além de outras literaturas de apoio (Sorgeloos et al., 1978; Parra, 2001; Pour \& Sasidharan, 2011; Batalini et al., 2020). Para a obtenção das larvas de A. salina, foram mantidos cistos em uma solução aquosa de sal marinho $0,037 \mathrm{~g} \cdot \mathrm{mL}^{-1}(\mathrm{~m} / \mathrm{v})$, sob aeração e iluminação constante com uma lâmpada de 40 W (em torno de $37-40^{\circ} \mathrm{C}$ ), por 48 horas até sua eclosão. Em seguida, foi preparado uma solução de concentração $5,0 \mathrm{mg} \cdot \mathrm{mL}^{-1}$, a partir da diluição de $125 \mathrm{mg}$ de cada amostra analisada em $25 \mathrm{~mL}$ de etanol PA. Desta solução padrão foram transferidos 25 $\mu \mathrm{L}, 50 \mu \mathrm{L}, 100 \mu \mathrm{L}, 300 \mu \mathrm{L}, 500 \mu \mathrm{L}, 700 \mu \mathrm{L}, 1000 \mu \mathrm{L}, 1300 \mu \mathrm{L}, 1600 \mu \mathrm{L}$ e $2000 \mu \mathrm{L}$ para tubos de ensaio, mantidos em estufa a $50^{\circ} \mathrm{C}$ até evaporação total do solvente. O procedimento foi realizado em triplicata. Após a evaporação total do solvente, foram adicionados a cada tubo $3 \mathrm{~mL}$ da solução salina contendo 10 larvas de A. salina e completado o volume do tubo de ensaio com a adição de solução salina até $5 \mathrm{~mL}$, obtendo-se as concentrações finais nos tubos de $25 \mu \mathrm{g} \cdot \mathrm{mL}^{-1}, 50 \mu \mathrm{g} \cdot \mathrm{mL}^{-1}, 100 \mu \mathrm{g} \cdot \mathrm{mL}^{-1}, 300$ $\mu \mathrm{g} . \mathrm{mL}^{-1}, 500 \mu \mathrm{g} \cdot \mathrm{mL}^{-1}, 700 \mu \mathrm{g} \cdot \mathrm{mL}^{-1}, 1000 \mu \mathrm{g} \cdot \mathrm{mL}^{-1}, 1300 \mu \mathrm{g} \cdot \mathrm{mL}^{-1}, 1600 \mu \mathrm{g} \cdot \mathrm{mL}^{-1}$ e $2000 \mu \mathrm{g} \cdot \mathrm{mL}^{-1}$ respectivamente. O tubo controle foi preparado contendo somente $5 \mathrm{~mL}$ de solução salina e 10 larvas de A. salina. Após 24 horas de exposição, com auxílio de lupa e pipeta Pasteur foi realizada a contagem do número de larvas sobreviventes, sendo consideradas mortas aquelas larvas que permanecerem imóveis por mais de 10 segundos após agitação branda dos tubos. Posteriormente foi realizado o cálculo da concentração letal média $\left(\mathrm{CL}_{50}\right)$ das amidas (I) a (V) utilizando o programa Statplus 2008 (Lhullier et al., 2006).

\section{Resultados e Discussões}

Na Figura 1 encontram-se as estruturas químicas das cinco amidas sintetizadas utilizadas neste estudo: 
Figura 1. Estruturas moleculares das amidas aromáticas sintetizadas utilizando método de Schotten-Baumann.<smiles>Cc1ccccc1NC(=O)c1ccccc1</smiles>

$N$-(2-metilfenil)-benzamida (I)<smiles>O=C(Nc1ccncc1)c1ccccc1</smiles>

$N$-(4-piridinil)-benzamida (III)

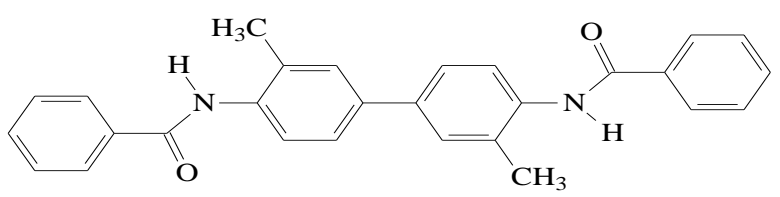

$N, N^{\prime}-\left(2,2^{\prime}\right.$-dimetil-4,4'- difenil)-dibenzamida (II)

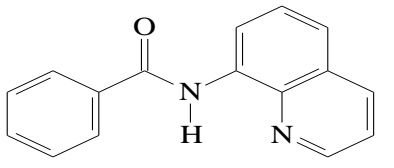

$N$-(8-quinolinil)-benzamida (IV)<smiles>O=C(O)c1ccc(NC(=O)c2ccccc2)cc1</smiles>

$N$-(4-carboxilfenil)-benzamida (V)

Fonte: Autores.

As amidas destacadas na Figura 1 foram escolhidas por apresentarem padrões de similaridade: todas são amidas aromáticas e secundárias. A substância (I) pode ser diretamente comparada com (II), em que ambas apresentam grupo metila doador de elétrons ao anel aromático; (III) e (IV) conferem um padrão semelhante com relação ao anel nitrogenoaromático piridil e quinolil, respectivamente; por fim, a substância (V), aromática, contém um grupo carboxila na posição 4, que exerce um efeito retirador de elétrons no anel aromático.

\subsection{Avaliação do potencial antioxidante das amidas (I) a (V) utilizando radical DPPH}

O ensaio usando DPPH baseia-se na capacidade desse composto em sequestrar radicais livres, sendo um teste crucial para analisar de forma qualitativa o nível de atividade antioxidante presente nas amidas estudadas e dessa forma viabilizar emprego das mesmas como materiais biológicos ou até mesmo compostos precursores para atividades de caráter bioativo (Celestino, 2011). A Tabela 1 a seguir demonstra os resultados para o fator de retenção (FR) e o padrão qualitativo do potencial antioxidante das cinco amidas analisadas.

Tabela 1. Resultados do perfil antioxidante qualitativo para as amidas (I) a (V) utilizando radical DPPH.

\begin{tabular}{c|c|c}
\hline AMOSTRA ANALISADA & $\begin{array}{c}\text { FATOR DE RETENÇÃO } \\
(\mathbf{F R})\end{array}$ & Forte \\
\hline$N$-(2-metilfenil)-benzamida (I) & 0,85 & Forte \\
\hline$N, N^{\prime}-\left(2,2^{\prime}\right.$-dimetil-4,4'-difenil)-dibenzamida (II) & 0,80 & Fraco \\
\hline$N$-(4-piridinil)-benzamida (III) & 0,75 & Fraco \\
\hline$N$-(8-quinolinil)-benzamida (IV) & 0,80 & Moderado \\
\hline$N$-(4-carboxilfenil)-benzamida (V) & 0,90 & \\
\hline
\end{tabular}

Fonte: Autores. 
Os valores encontrados de FR para as amidas, no eluente utilizado, encontraram-se todos acima de 0,5. Nesta análise, as amidas (I), (II) e (V) apresentaram atividade antioxidante de forte e moderado, podendo se caracterizar como compostos viáveis para emprego bioativo. A forte atividade antioxidante revelada para as amidas (I) e (II) devem estar associados à presença de grupo doador de elétrons (metila) no anel aromático; já para a amida (V), a presença de grupo retirador de elétrons (carboxila) parece estar associado à diminuição dessa atividade. A fraca atividade antioxidante encontrada para as amidas estruturalmente semelhantes (III) e (IV) deve estar relacionada, finalmente, com a presença de aneis heterocíclicos nitrogenados, que deve inibir a possibilidade da substância atuar como bom capturador de radicais livres.

\subsection{Avaliação do potencial tóxico das amidas (I) a (V) frente às larvas de Artemia salina Leach}

O bioensaio com Artemia salina possibilita estabelecer valores para taxa de letalidade através do CL $_{50}$ - concentração média letal. Essa análise é importante pois estima a potência de ação de cada amida frente aos microcrustáceos e estabelece o nível de toxicidade de cada uma delas (Harada, 2009). A literatura preconiza que os valores de CL50 (Concentração Letal Média) para testes com A. salina que forem menor que $100 \mu \mathrm{g} . \mathrm{mL}^{-1}$ indicam substâncias tóxicas, entre 100 e $500 \mu \mathrm{g} . \mathrm{mL}^{-1}$ moderadamente tóxicas, entre 500 e $1000 \mu \mathrm{g} \cdot \mathrm{mL}^{-1}$ fracamente tóxicas e maior que $1000 \mu \mathrm{g} \cdot \mathrm{mL}^{-1}$ atóxicas (Nguta et al., 2011). A Tabela 2 demonstra os valores de concentração média letal CL 50 para cada amostra testada bem como o padrão encontrado segundo consta a literatura. Na sequência (Figuras 2 a 6) são apresentados os gráficos produzidos através do programa Statplus.

Tabela 2. Resultados de $\mathrm{CL}_{50}$ para as análises de toxicidade das amidas aromáticas (I) a (V).

\begin{tabular}{c|c|c}
\hline AMOSTRA ANALISADA & CL50 $\left(\boldsymbol{\mu g} \cdot \mathbf{m L}^{-\mathbf{1}}\right)$ & $\begin{array}{c}\text { PADRÃO ENCONTRADO } \\
\text { (NGUTA et al., 2011) }\end{array}$ \\
\hline$N$-(2-metilfenil)-benzamida (I) & 347,1 & Moderadamente tóxico \\
\hline$N, N^{\prime}-\left(2,2^{\prime}\right.$-dimetil-4,4'-difenil)-dibenzamida (II) & 667,0 & Fracamente tóxico \\
\hline$N$-(4-piridinil)-benzamida (III) & 662,5 & Tóxico \\
\hline$N$-(8-quinolinil)-benzamida (IV) & 4,4 & Atóxico tóxico \\
\hline$N$-(4-carboxilfenil)-benzamida (V) & 1128,7 & \\
\hline
\end{tabular}

Fonte: Autores.

Figura 2. Gráfico de $\mathrm{CL}_{50}$ para a amida $N$-(2-metilfenil)benzamida (I).

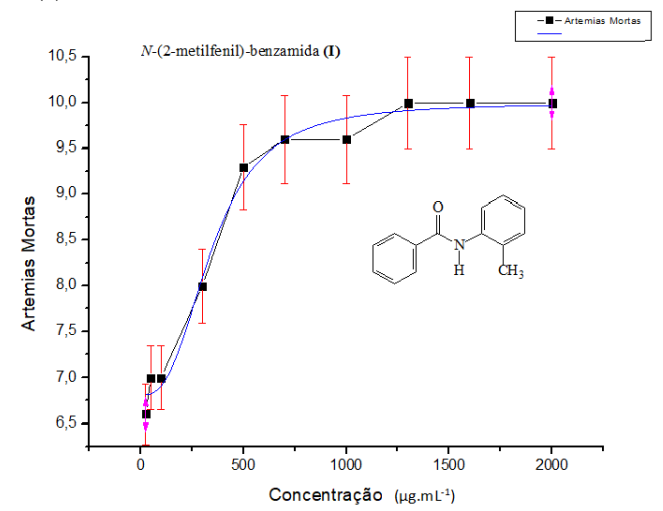

Fonte: Autores.
Figura 3. Gráfico de $\mathrm{CL}_{50}$ para a amida $N, N^{\prime}-(2,2$ 'dimetil-4,4'-difenil)-dibenzamida (II).

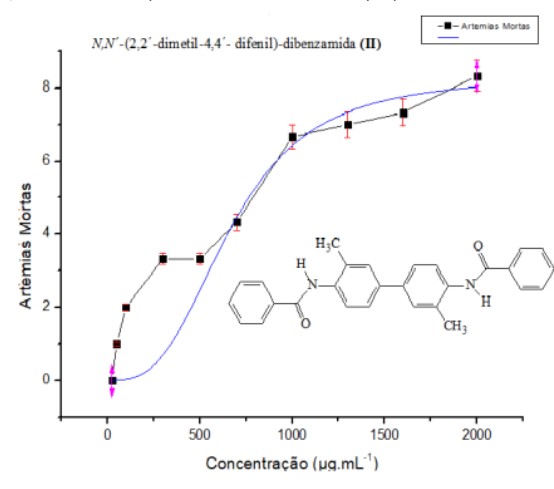

Fonte: Autores. 

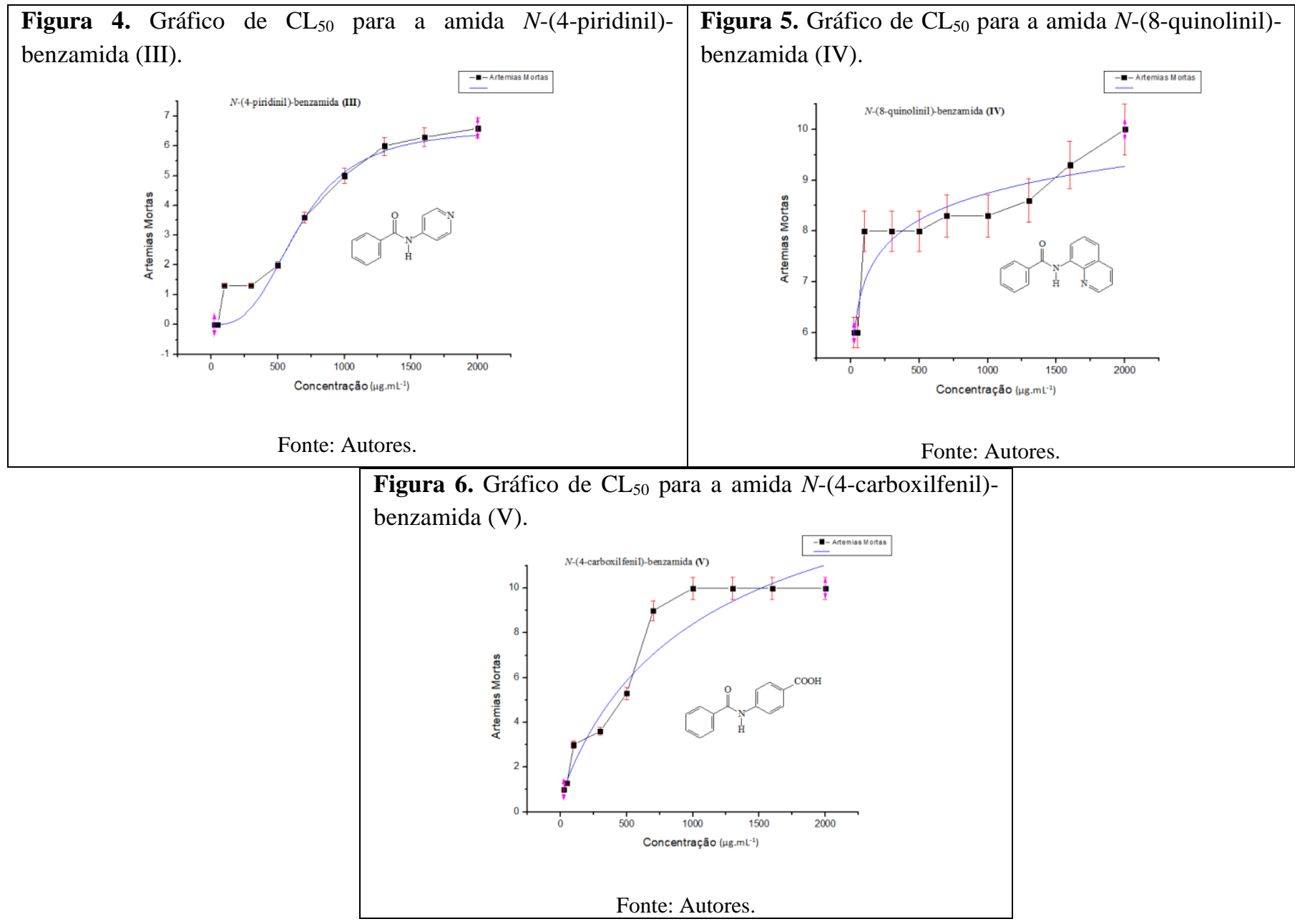

De acordo com os valores encontrados de $\mathrm{CL}_{50}$ (Tabela 2) e partindo do princípio de análise de relação estruturaatividade, entende-se que $N$-8-quinolinilbenzamida (IV) apresenta um anel benzênico a mais em sua estrutura quando comparado à amida (III), que teve perfil fracamente tóxico para esse bioensaio, e desta maneira o benzeno extra em sua estrutura química poderia justificar certas características para o perfil de toxicidade elucidado. As amidas (I) e (II), que apresentam grupo metila doador de elétrons, exibiram padrões próximos de toxicidade, que vão de moderadamente a fracamente tóxicos. Já a substância $(\mathrm{V}), N$-4-carboxilfenilbenzamida, que apresentou perfil atóxico, pode ter a explicação baseado na presença do grupamento carboxila $(-\mathrm{COOH})$, retirador de elétrons do anel benzênico. Em linhas gerais, esse teste sinaliza que principalmente as amidas (II), (III) e (V) apresentam a possibilidade de aplicabilidade para uso seguro como desenvolvimento de possível fármaco, enquanto que a amida (I) - moderadamente tóxica e (IV) - tóxica, não seriam recomendadas para efeito bioativo pois apresentam valores de $\mathrm{CL}_{50}$ consideráveis para o não uso seguro em seres vivos.

\section{Considerações Finais}

A síntese verde realizada pelo método de Schotten-Baumann demonstrou ser uma opção viável para a produção de novas amidas aromáticas, de modo a garantir o elo entre a cadeia de produção industrial e o cuidado com o meio ambiente, isto é, sem agressão, poucos resíduos, baixo gasto energético e de matéria-prima química.

Os resultados obtidos de atividade antioxidante sinalizam eleger as amidas (I) e (II) como as que melhor podem atuar no sentido de capturar radicais livres do meio, sendo essa atividade tangenciada pela amida (V). Já com relação ao uso seguro, 
os resultados encontrados de $\mathrm{CL}_{50}$ para as substâncias analisadas apontam três delas: $N, N^{\prime}$-(2,2'-dimetil-4,4'-difenil)dibenzamida (II), $N$-(4-piridinil)-benzamida (III) e $N$-4-carboxilfenilbenzamida (V).

As amidas (II) e (V) ainda não possuem registros na literatura até o presente momento, podendo então serem consideradas estruturas químicas inéditas. Ainda cabem estudos futuros para condicionar o uso seguro dessas três amidas, porém o mais crucial dessa pesquisa é demonstrar que a boa junção entre a síntese orgânica e a química verde é possível, eficaz e deve ser cada mais adotada pelas indústrias químicas.

O grupo de pesquisa planeja estudos futuros a serem conduzidos com as substâncias desse estudo, no sentido de testar seus potenciais tóxicos frente à outros modelos celulares, bem como investigações de outras atividades biológicas, como por exemplo antifúngica, antibacteriana e antitumoral.

\section{Agradecimentos}

À UFMT - Campus Universitário do Araguaia e aos membros pesquisadores do LAPQUím - Laboratório de Pesquisas em Química de Produtos Naturais - UFMT/CUA.

\section{Referências}

Anastas, P. T. \& Eghbali, N. (2010). Green Chemistry: Principles and Practice. Chemical Society Reviews, 39 (1), $301-312$.

Barbosa, K. B. F., Costa, N. M. B., Alfenas, R. C. G., Paula, S. O., Minin, V. P. R. \& Bressan, J. (2010). Estresse Oxidativo: conceito, implicações e fatores modulatórios. Revista de Nutrição, 23 (4), 629-643.

Barreiro, E. J. \& Fraga, C. A. M. (2015). Química medicinal: as bases moleculares da ação dos fármacos. (3a ed.), Artmed 608 p.

Batalini, C., Stocco, L. O., Fernandes, R. T. S. \& Junior, J. M. (2020). Avaliações fitoquímica, fitotóxica e antifúngica da entrecasca do caule de Pterodon pubescens Benth (sucupira branca). Brazilian Journal of Development, 6 (10), 77589-77607.

Baumann, E. (1886). Ueber eine einfache methode der darstellung von benzoësäureäthern. Berichte der Deutschen Chemischen Gesellschaft, 19 (2), 32183222 .

Calvalcante, P. M. M., Silva, R. L., Freitas, J. J. R., Freitas, J. C. R. \& Filho, J. R. F. (2015). Proposta de preparação e caracterização de ésteres: um experimento de análise orgânica na graduação. Educación Química, 26 (4), 319-329.

Celestino, M. T. (2011). Estudo do uso racional de antioxidantes em formulações de sólidos orais. Dissertação de Mestrado em Ciências Farmacêuticas, Universidade Federal do Rio de Janeiro, Faculdade de Farmácia, UFRJ, Rio de Janeiro.

Harada, T. N. (2009). Correlações entre ensaios de citotoxidade em Artemia salina Leach e atividade antineoplásica sobre linhagens de células tumorais para algumas classes de produtos naturais. Dissertação de Mestrado apresentada ao Programa de Pós-Graduação em Saúde e Desenvolvimento da Região Centrooeste, Universidade Federal de Mato Grosso do Sul, UFMS, Campo Grande - MS.

Lenardão, E. J., Freitag, R. A., Dabdoub, M. J., Batista, A. C. F. \& Silveira, C. C. (2003). "Green chemistry” - Os 12 princípios da química verde e sua inserção nas atividades de ensino e pesquisa. Química Nova, 26 (1), 123-129.

Lhullier, C., Horta, P. \& Falkenberg, M. (2006). Avaliação de extratos de macroalgas bênticas do litoral catarinense utilizando o teste de letalidade para Artemia salina. Revista Brasileira de Farmacognosia, 16 (2), 158-163.

Mãrino, P. A. (2014). Estudo de Chalconas como antibacterianos Potenciais: Síntese, Avaliação da Ação Antibacteriana e das Propriedades Físico-Químicas. Dissertação de Mestrado em Ciências Farmacêuticas, Programa de Pós-Graduação em Ciências Farmacêuticas, Universidade Federal do Pampa, Uruguaiana.

Medeiros, H. I. R., Silva, B. B. M., Aguiar, C. E. R., Brito, T. A. M., Ferreira, F. E. S., Fernandes, N. D., Moura, E. P. \& Júnior, F. C. M. (2020). Síntese, elucidação da arquitetura molecular e avaliação do potencial citotóxico de um promissor candidato a fármaco derivado de amidoxima. Brazilian Journal of Development, 6 (9), 66070-66079.

Meyer, B. N., Ferrigni, N. R., Putnam, J. E., Jacobsen, L. B., Nichols, D. E. \& Mclaughlin, J. L. (1982). Brine shrimp, a convenient general bioassay for active-plant constituents. Planta Medica, 45 (1), 31-34.

Nguta, J. M., Mbaria, J. M., Gakuya, D. W., Gathumbi, P. K., Kabasa, J. D. \& Kiama, S. G. (2011). Biological screening of Kenya medicinal plants using Artemia salina L. (Artemiidae). Pharmacology on line, 2, 458-78.

Parra, A. L., Sardiñas, I. G., Yhebra, R. S. \& Buelba, L. I. (2001). Comparative study of the assay of Artemia salina Leach and the estimate of the medium lethal dose $\left(\mathrm{LD}_{50}\right.$ value) in mice, to determine oral acute toxicity of plant extracts. Phytomedicine, 8 (5), 395-400.

Pour, B. M. \& Sasidharan, S. (2011). In vivo toxicity study of Lantana camara. Asian Pacific Journal of Tropical Biomedicine, 1 (3), $230-232$. 
Research, Society and Development, v. 11, n. 2, e58811225913, 2022

(CC BY 4.0) | ISSN 2525-3409 | DOI: http://dx.doi.org/10.33448/rsd-v11i2.25913

Rockenbach, I. I. (2008). Compostos fenólicos, ácidos graxos e capacidade antioxidante do bagaço da vinificação de uvas tintas (Vitis vinifera L. e Vitis labrusca L.). Dissertação de Mestrado em Ciência dos Alimentos, Centro de Ciências Agrárias, Universidade Federal de Santa Catarina.

Schotten, C. (1884). Ueber die oxydation des piperidins. Berichte der Deutschen Chemischen Gesellschaft, 17 (2), 2544-2547.

Solomons, G., Fryhle, C. \& Snyder, S. (2014). Organic Chemistry. (11a ed.), John Wiley \& Sons, 648 p.

Sorgeloos, P., Van Der Wielen, C. R. \& Persoone, G. (1978). The use of Artemia nauplii for toxicity tests. A critical analysis. Ecotoxicology and Environmental Safety, 2, (3-4), 249-255.

Sousa, C. M. M., Rocha e Silva, H., Vieira Jr, G. M., Ayres, M. C. C., Costa, C. L. S., Araújo, D. S., Cavalcante, L. C. D., Barros, E. D. S., Araújo, P. B. M., Brandão, M. S. \& Chaves, M. H. (2007). Fenóis totais e atividade antioxidante de cinco plantas medicinais. Química Nova, 30 (2), 351-355.

Zimmerman, J. B., Anastas, P. T. \& Erythropel, H. C. \& Leitner, W. (2020). Designing for a green chemistry future. Science, 367 (6476), $397-400$. 